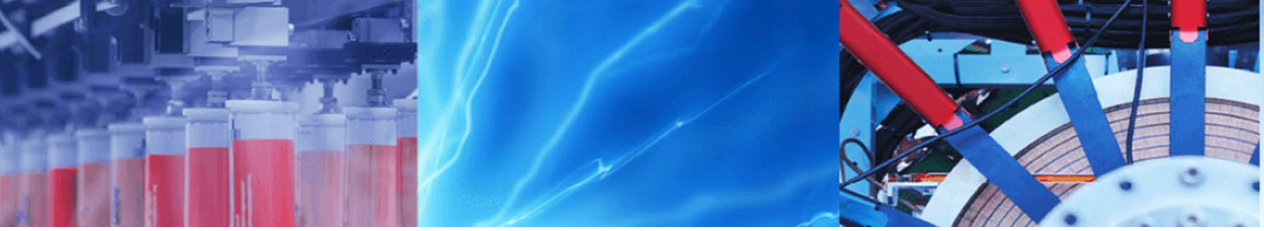

Research Article

\title{
A robust technique of fake news detection using Ensemble Voting Classifier and comparison with other classifiers
}

\author{
Atik Mahabub $^{1}$ (D)
}

Received: 8 December 2019 / Accepted: 25 February 2020 / Published online: 2 March 2020

(c) Springer Nature Switzerland AG 2020

\begin{abstract}
These days online networking is generally utilized as the wellspring of data as a result of its ease, simple to get to nature. In any case, expending news from online life is a twofold edged sword as a result of the widespread of fake news, i.e., news with purposefully false data. Fake news is a major issue since it affects people just as society substantial. In the internet based life, the data is spread quick and subsequently discovery component ought to almost certainly foresee news quick enough to stop the dispersal of fake news. Consequently, identifying fake news via web-based networking media is a critical and furthermore an in fact testing issue. In this paper, Ensemble Voting Classifier based, an intelligent detection system is proposed to deal with news classification both real and fake tasks. Here, eleven mostly well-known machine-learning algorithms like Naïve Bayes, K-NN, SVM, Random Forest, Artificial Neural Network, Logistic Regression, Gradient Boosting, Ada Boosting, etc. are used for detection. After cross-validation, we used the best three machinelearning algorithms in Ensemble Voting Classifier. The experimental outcomes affirm that the proposed framework can accomplish to about $94.5 \%$ outcomes as far as accuracy. The other parameters like ROC score, precision, recall and F1 are also outstanding. The proposed recognition framework can effectively find the most important highlights of the news. These can also be implemented in other classification techniques to detect fake profiles, fake message, etc.
\end{abstract}

Keywords Fake news · Voting classifier $\cdot$ Machine learning $\cdot$ Data mining

\section{Introduction}

Almost all people confront misleading conduct in our everyday life. Individuals mislead escape from a circumstance that appears to be negative to them. As a result, a few untruths are harmless however others may have extreme repercussions in the general public. Reports recommend that the capacity of people to recognize misleading without uncommon guides is just 54\% [1].

An investigation by DePaulo et al. [2]. discovered that trickiness with no specific inspiration or aim showed no discernible signals of double-dealing. Be that as it may, prompts were essentially more when lies were about transgressions. With the ascent in the number of criminal cases documented each year in the US, it is morally and ethically imperative to denounce just the blameworthy respondent and free the guiltless. Since the judgment for any case is for the most part dependent on the hearings and proof from the partners (denounced, witnesses, and so forth.), the judgment is well on the way to turn out badly if the partners don't talk reality.

It is, thus, imperative to recognize tricky conduct precisely so as to upkeep the lawfulness. Internet-based life can be described as a virtual existence where individuals collaborate with one another without the human feel and contact. It is anything but difficult to not uncover one's character as well as profess to be another person on the internet based life. Cyberbullying is progressively

Atik Mahabub, atikmahabub1209042@gmail.com | 1 Department of Electronics and Communication Engineering, Khulna University of Engineering \& Technology, Khulna 9203, Bangladesh.

SN Applied Sciences (2020) 2:525 | https://doi.org/10.1007/s42452-020-2326-y 
turning into a typical issue among adolescents these days [3]. These incorporate spreading gossipy tidbits about an individual, dangers, and inappropriate behavior. Cyberbullying unfavorably influences the person in question and prompts an assortment of passionate reactions, for example, brought down confidence, expanded self-destructive considerations, outrage, and wretchedness [4]. Youngsters fall prey to these assaults because of their failure to grasp the sophistry and self-absorbed conduct of the aggressor. Another territory where a misleading location is of central significance is with the expanded number of false stories, a.k.a Fake News, on the Internet. Late reports recommend that the result of the U.S. Presidential Elections is because of the ascent of online phony news [5]. Advocates use contentions that, while now and again persuading, are not really legitimate. Web-based life, for example, Facebook and Twitter, have turned into the propellers for this political purposeful publicity. Nations around the globe, for example, France, are utilizing strategies that would keep the spread of phony news amid their decisions [5]. Despite the fact that these measures may help, there is a squeezing requirement for the computational phonetics network to devise productive strategies to battle Fake News given that people are poor at distinguishing double-dealing. Hence, it is in extraordinary need of a programmed indicator to relieve the genuine negative effects brought about by the fake news [6]. There are many methodology such as correlation filter based tracking algorithms [7], non-negative least square algorithm [8], Online Representative Sample Selection method [9], regularization framework [10], multiple feature fused model [11] have been introduced.

The whole work is presented in four sections as follows. Segment 2 describes the related works in the field of fake news detection. A review about likelihood classifications and algorithm are talked about in Segment 3. Segment 4 presents the experimental results. In this part, further discussions and analyzations are also presented. Segment 5 is the brief summary of this work and the blueprint of the future works.

\section{Literature review}

There are numerous errands identified with fake news recognition, for example, rumor discovery [12] and spam detection [13]. Following the past work $[14,15]$, we indicate the meaning of fake news as news which is deliberately created and can be confirmed as false. In fake news identification errand, the principle challenge is the means by which to recognize news as indicated by highlights. The highlights can be extricated from posts, social setting, and even appended pictures.
Fake news recognition has pulled in light of a legitimate concern for specialists as of late and a few methodologies have been proposed. As of late there are takes a shot at utilizing content substance of the news for the identification undertaking $[16,17]$. Wang [18] utilizes CNN for the order of fake news content. Shu et al. $[16,17]$ utilize the dormant substance installing of the record as one of the highlights for the recognition undertaking. There are a few different works which make utilization of content substance.

Ruchansky et al. [19] utilize social commitment at the post level to catch the distinctions in transient commitment designs among phony and genuine news. Since individuals express their feelings towards news through web-based social networking post thus it is sensible to utilize web-based life posts as a potential component for highlight location. Shu et al. [20,21] utilize different highlights of the client drawing in with the news articles to recognize the fake news.

In this paper, we will work on mostly used ML algorithms to choose the best classification algorithms. Among them, the three best performers will be utilized in the Voting Classifier. From this, we can best accurate result from these classifiers. Our work provides more accurate results compared with the other works.

\section{Methodology}

The proposed architecture can be divided into many subsections. The flowchart of the work can be seen from Fig. 1 .

\subsection{Data collection}

Firstly, a Data Set is needed with fake and real news. The proposed system is tested on the Data Set of 6500 data from which about 3252 data are fake and 3259 data are real which has been used by Wang [16]. It is a Data Set of combination of real and fake news.

\subsection{Preprocessing}

In all actuality data index, which contains various missteps, they are refreshed and removed in order to have definite results in the data index. In this movement data collection, it is changed and composed into a legitimate plan before classifiers are associated in the data index. The record has suitably taken care of before classifiers are associated on it. The Dataset is mostly on the English Language. For preprocessing the data natural language processing (NLP) technique is applied where we take only English words. It helps to improve accuracy. After that, text transformation and binaries into the data set are performed to ease the data preprocessing. 


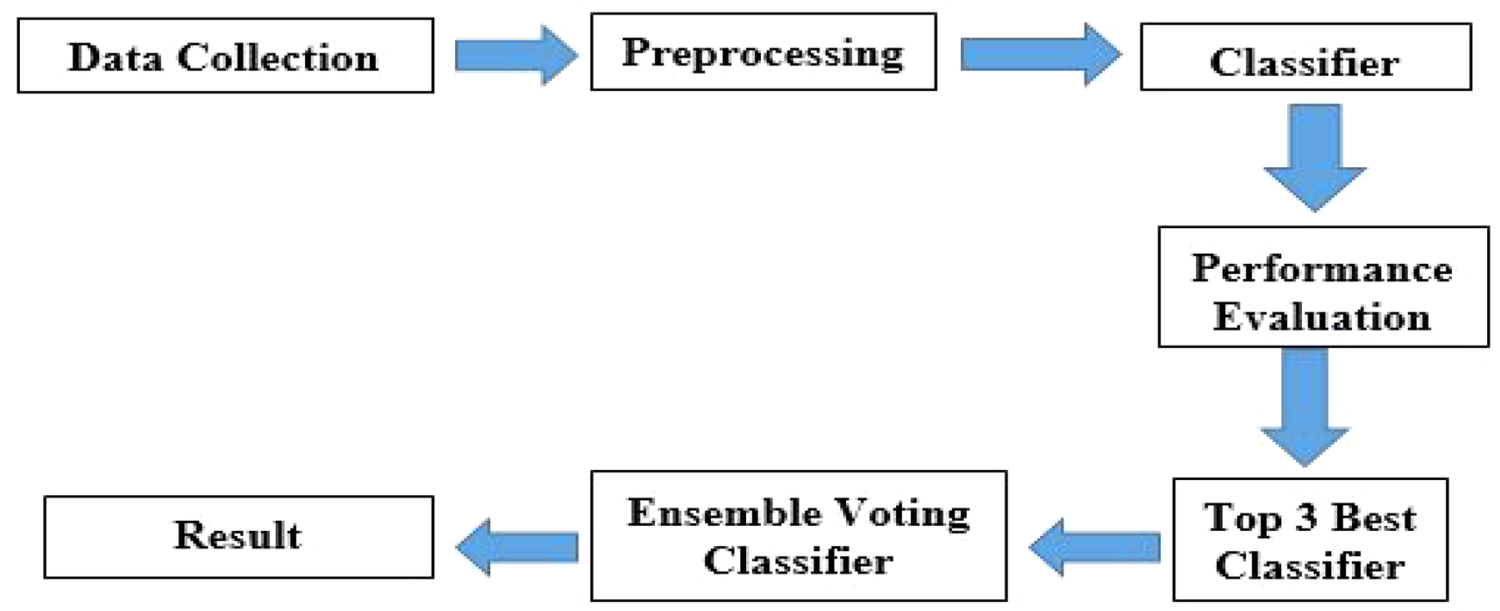

Fig. 1 Flowchart of the ensemble architecture

\subsection{Classifier}

Subsequent to having the preprocessed document, all the known classifier, in particularly, Support Vector, K-Nearest Neighbors, Ada Boost, Naïve Bayesian, Neural Network, Decision Tree, Gradient Boosting, Extreme Gradient Boosting, Random Forest, Logistic Regression, etc. have been applied so as to discover includes based on which fake being detected.

\subsection{Performance evaluation}

For the performance evaluation, cross validation technique has been utilized. Here the K-fold cross validation has been performed. Accordingly, the dataset is divided into $10 \mathrm{~K}$-fold [22].

In implementation of this step, model_selection function of scikit-learn has been used. Stratified K-Fold subfunction has been used to split the training dataset in K-fold for cross-validation, cross_val_score sub-function has been used to observe the cross-validation scores of ML classifiers and GridSearchCV sub-function has been used to hyper-tune the ML classifiers.

Resulting to applying all classifiers, all of them was surveyed dependent on execution estimations Like test score, ROC score, precision score, recall value and so forth in order to comprehend the best classifier.

\subsection{Choosing top 3 classifier}

After the performance assessment of the diverse traditional used ML classifiers, the best three best classifiers have been recognized. At that point, these main three classifiers will be used for the next step to tune to obtain the best output from the data set. Then the Voting classifier will be utilized.

\subsection{Utilizing Ensemble Voting Classifier}

For the Ensemble Classifier, here this article will talk about the Voting Classifier. Top three classifier will be used for this Voting Classification to get the best execution and output.

\subsection{Results}

At the last advance, the presentation of the Voting Classifier will be surveyed dependent on execution estimations Like test score, ROC score, precision score, recall value and so forth. The outcomes at that point will be contrasted and other important works for assessing the outcomes.

\subsection{Ensemble Voting Classifier}

The Ensemble Voting Classifier $[23,24]$ is a meta classifier for uniting similar or skillfully unprecedented machine learning classifiers for classification and detection. The Ensemble Voting Classifier executes "hard" and "soft" voting.

\subsection{Hard voting}

In the hard ensemble, voting is the most effortless instance of the greater part of voting. Here, we would determine the classmark $Y$ through lion's share voting of each classifier C: 
$\mathrm{Y}=\operatorname{mode}\{\mathrm{C} 1(\mathrm{x}), \mathrm{C} 2(\mathrm{x}), \ldots, \mathrm{Cm}(\mathrm{x})\}$

\subsection{Soft voting}

In soft ensemble voting, we envision the class names subject to the foreseen probabilities $p$ for the classifier, this procedure is perhaps recommended if the classifiers are particularly adjusted.

$Y=\operatorname{argmax}_{i} \sum_{(j=1)}^{m} W_{j} P_{i j}, \quad \mathbf{i} \in\{\mathbf{0}, \mathbf{1}\}, \quad[\mathrm{j}=1,2, \ldots, \mathrm{m}]$

where $\mathrm{Wj}$ is the heap that can be doled out to the jth classifier.

The brief discussions of some classifiers which have been used for selecting the top three classifiers for the ensemble are below:

\subsection{Naïve Bayesian classifier}

It was one of the primary characterization procedures utilized for fake news detection. It takes a shot at Bayes Theorem of likelihood to check if the approaching news is a fake or not. The filter in this classifier initially must be prepared to check for fake news. Preparing a data collection implies that the channel is given a lot of words the client gives by physically recognizing the news as fake or not. Through the contribution from a client, the classifier is currently prepared and can browse approaching news. This classifier checks the likelihood of the words in the preparation set in approaching news and with the outcome acquired it can channel fake news. It makes an alternate organizer for the fake and moves those news straightforwardly to the folder. In spite of the fact that it is entirely old, it is as yet favored over increasingly refined classifiers [25].

\subsection{K-Nearest Neighbor}

In this procedure of classification, a training set contains an example of news which can assist in distinguishing whether the approaching news is fake or not. The approaching news is contrasted with the training set with discovering its k-closest part found by contrasting it and the training set and its k most comparable archive is found and after that recognized as fake, in light of which bunch its k most comparative report was found [26].

\subsection{Support Vector Machine classifier}

In this technique, a choice plane is shaped to isolate fake and genuine news. They are isolated by a choice limit which has certain conditions to isolate this news. A training set is framed for the arranging and approaching news is contrasted and the preparation set. Like k-closest, neighbor the approaching news is contrasted with the preparation set with discover similitude between the approaching news and training set. A part work, $\mathrm{K}$ is utilized to decide the closeness and dependent on this the news are arranged in the choice plane [27].

\subsection{Artificial Neural Network}

Artificial Neural Network acts as a fake human mind. Fake neurons are interconnected to frame this system and information is gone through it for learning. Like the human mind, it learns by precedent and amid preparing, the information is gone through the system so it can learn and adjust as per the models [28]. They change their structure dependent on data from the models with the goal that a superior arrangement of grouping can be shaped [29].

\subsection{Decision Tree classifier}

Decision Tree is a prescient displaying approach which is utilized in machine learning, data mining, and insights. It makes a model based on a few information factors predicts the estimation of an objective variable. It is a broadly utilized calculation which pursues the ravenous methodology at each split and dynamically fabricates a tree. Every hub of a Decision Tree speaks to analysis on quality, branches speak to the aftereffect of the examinations and the leaf hubs contains the class marks. The choice tree parts are picked with the end goal that they limit pollution and expand immaculateness of the subset being developed [30].

\subsection{Random Forest classifier}

It is also a tree-based classification. An arrangement is performed by creating various distinctive Decision Trees, every one of which has an alternate component structure. From that point forward, a class is appointed dependent on the greater part votes of the distinctive trees [17].

\subsection{Ada Boosting classifier}

An Ada Boost Classifier is a meta predictor that starts by fitting a classifier on the first dataset and afterward fits extra duplicates of the classifier on the equivalent dataset yet where loads of inaccurately grouped examples are balanced with the end goal that consequent classifiers center more around troublesome cases [31]. 


\subsection{Gradient Boosting classifier}

Gradient Boosting is a machine learning system for relapse and classification issues, which creates a forecast show as a gathering of feeble expectation models, normally Decision Trees. It fabricates the model in a phase astute design like other boosting strategies do, and it sums them up by permitting enhancement of a discretionary differentiable misfortune work [32].

\subsection{Logistic Regression classifier}

Logistic Regression is evaluating the parameters of a Logistic model; it is a type of binomial relapse. Scientifically, a twofold calculated model has a reliant variable with two

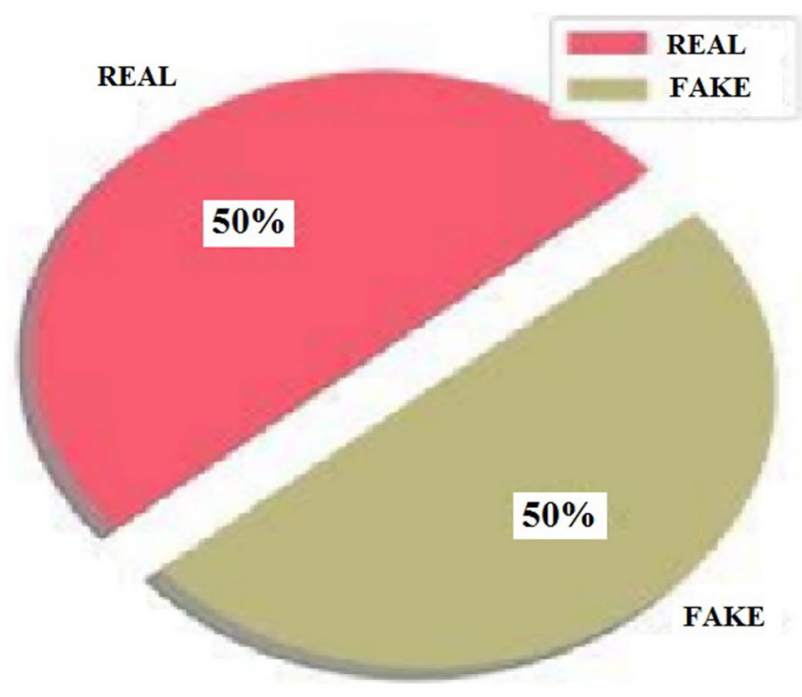

Fig. 2 The pie chart representation of dataset conceivable qualities, for example, pass/fail, win/lose, alive/dead; these are spoken to by a pointer variable, where the two qualities are marked " 0 " and " $1 "$. In the Logistic model, the log-chances for the esteem named "1" is a direct blend of at least one autonomous factors; the free factors can each be a double factor or a constant variable. The relating likelihood of the esteem marked "1" can differ somewhere in the range of 0 and 1, henceforth the naming; the capacity that changes over log-chances to likelihood is the strategic capacity, consequently the name [33].

\section{Result analysis}

From the collected dataset contained about 6500 news from which about 3252 news is fake and 3259 news is real which has been referred in Data Collection of Methodology. The representation of the Pie Chart of this Data set has been provided in Fig. 2 . The dataset which is used in this work is verified and analyzed with eleven different Machine Learning classification algorithm techniques that are used in cross-validation which are the following: (a) Random Forest, (b) Ada Boosting, (c) Gradient Boosting, (d) Extra Trees, (e) Logistic Regression, (f) K-Neighbors, (g) Decision Tree, (h) Multinomial Naïve Bayes, (i) MultiLayer Perception, (MLP), (j) Support Vector Machine and (k) Extreme Gradient Boosting. After preprocessing the dataset like cleaning the missing values, vector transforming the data, etc., the training data is split into tenfolds. After that, we measure the cross-validation score of these eleven classifiers. Figure 3 and Table 1 represents the crossvalidation score of these eleven classifiers.

After the result of the cross-validation scores, we choose the best three ML classification algorithms (1) MLP, (2)
Fig. 3 Cross-validation score representation of several ML classifiers by using bar chart

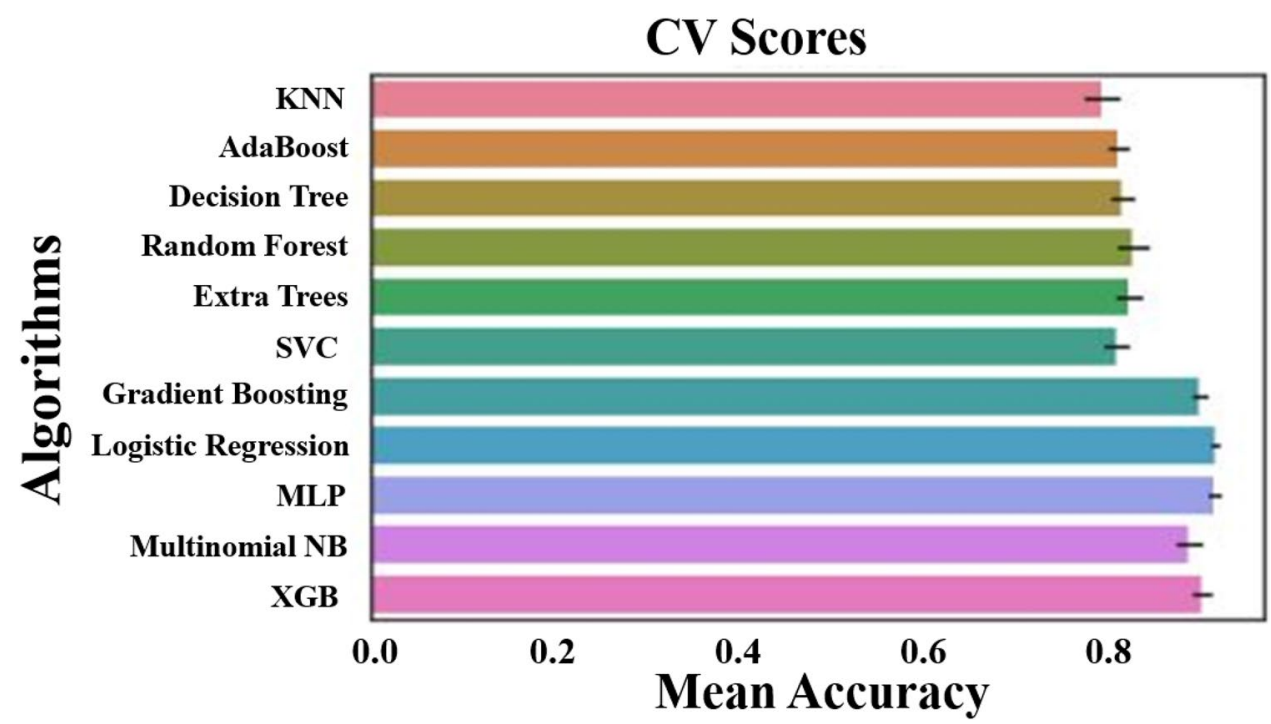

SN Applied Sciences A SPRINGER NATURE journa 
Table 1 Cross validation score of several ML classifiers

\begin{tabular}{ll}
\hline Classification type & $\begin{array}{l}\text { Cross valida- } \\
\text { tion score (\%) }\end{array}$ \\
\hline K-Neighbors & 79.63 \\
Ada Boost & 82.62 \\
Decision Tree & 82.69 \\
Random Forest & 83.59 \\
Extra Tree & 82.48 \\
SVC & 84.75 \\
Gradient Boosting & 90.30 \\
Logistic Regression & 91.97 \\
Multi-Layer Perception (MLP) & 91.9 \\
Multinomial Naïve Bayes & 89.17 \\
X-Gradient Boosting & 90.53 \\
\hline
\end{tabular}

Logistic Regression and (3) X-Gradient Boosting for the next step. Here, we will hyper-tune these three classifiers to get the best results from them (Fig. 4).

For MLP classification, we tune the parameters based on alpha, hidden layer size, maximum iterations, solver and random state. After tuning the parameter, we get best results for these parameters: 'alpha': 0.01, 'hidden layer sizes': 14, 'maximum iteration': 100, 'random state': 0 , 'solver': 'Ibfgs'. For MLP, the best score 92.59 , accuracy 93.83, precision 85.94 , recall 85.49 and ROC score 93.83 (Fig. 5).

For X-Gradient Boosting classification, we tune the parameters based on gamma, learning rate, loss, maximum depth, minimum sample leaf and $n \_$estimators. After tuning the parameter, we get best results for these

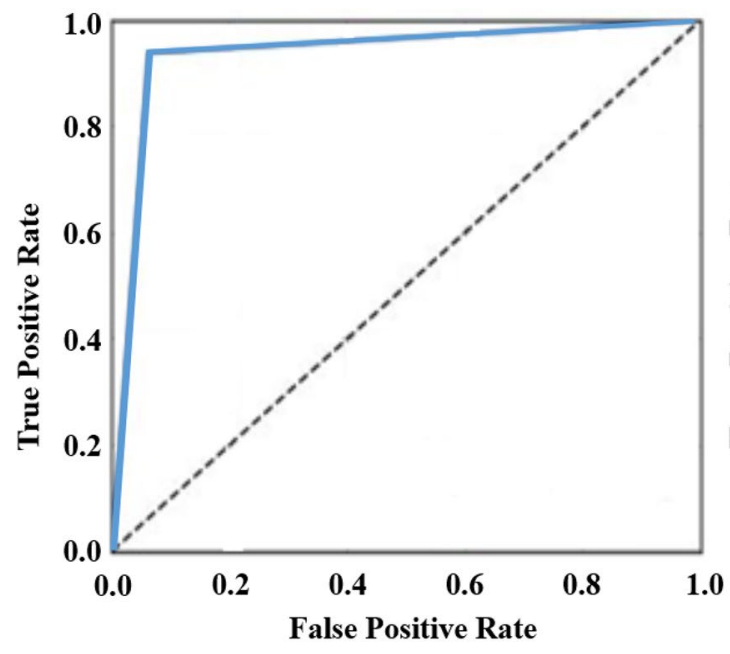

(a)

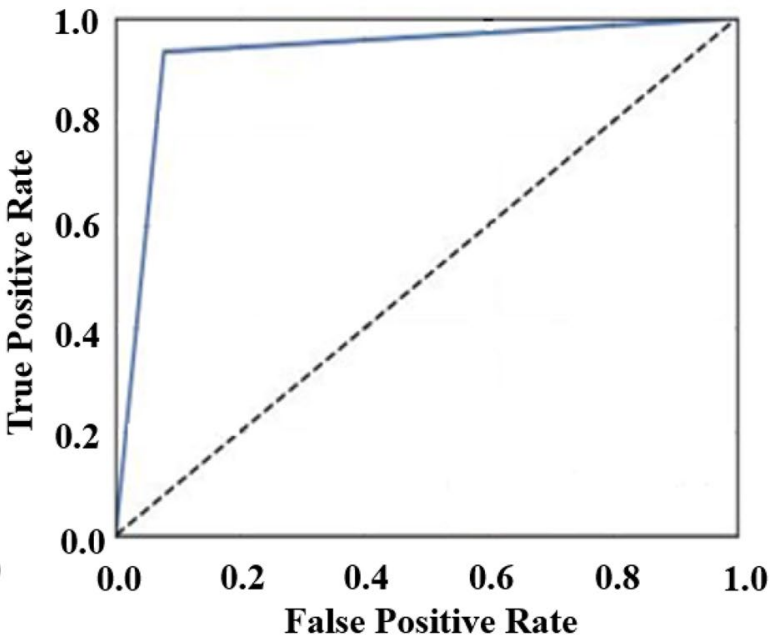

(b)

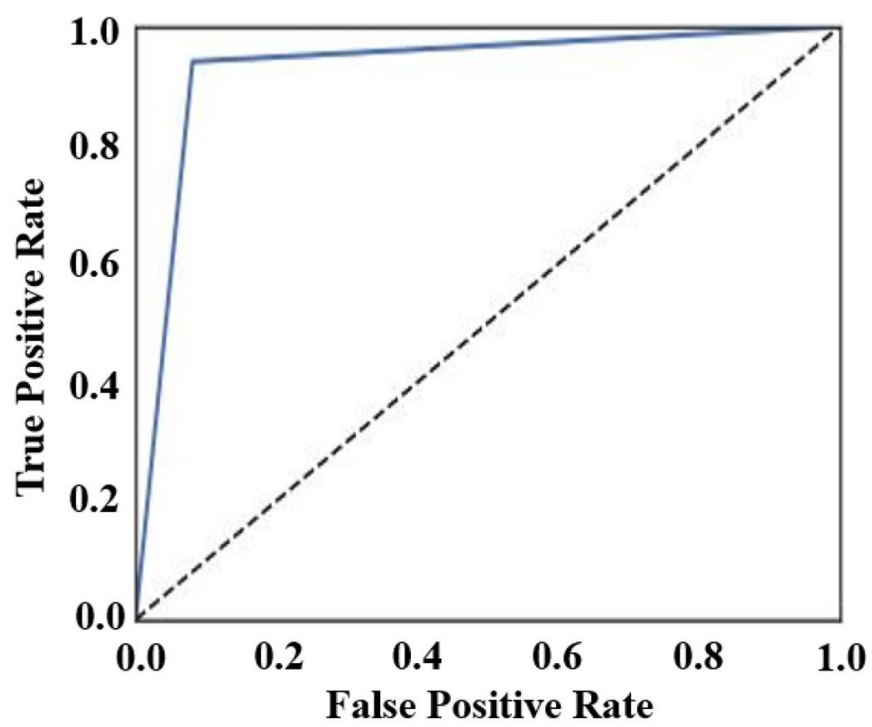

(c)

Fig. 4 ROC curve of a MLP, b X-Gradient Boosting and c Logistic Regression 
parameters: 'gamma': 1, 'learning rate': 0.1, 'loss': 'deviance', 'maximum depth': 15, 'min samples leaf': 5, 'n_estimators': 100. For X-Gradient Boosting, the best score 92.92, accuracy 92.87 , precision 86.67 , recall 87.67 and $R O C$ score 92.87.

For Logistic Regression classification, we tune the parameters based on tolerance, maximum iteration, $C$, intercept scaling, penalty and solver. After tuning the parameter, we get best results for these parameters: ' $C$ ': 0.1 , 'intercept scaling': 1, 'maximum iteration': 100, 'penalty': 'I2', 'solver': 'liblinear', 'tolerance': 0.0001 . For Logistic Regression, the best score 92.57 , accuracy 93.03 , precision 85.44, recall 90.01 and ROC score 93.03 (Table 2).

After receiving the best results from these three ML classification algorithm, we will use these in voting classifier to get a maximum test score. The ultimate test score of Ensemble Soft Voting Classifier is 94.47 . The other parameters of this classifier are Precision 95, Recall 95, F1 95 and ROC score 94.49. On the other hand, The ultimate test score of Ensemble Hard Voting Classifier is 93.99. The other parameters of this classifier are Precision 94, Recall 94, F1 94 and ROC score 93.98 (Table 3).

From the analysis, it is clear that Ensemble Voting Classifier provides best Test Score from all individual ML classification algorithms.

\section{Conclusions}

In this research work, a unique multi-classifier based Ensemble Voting Classifier technique is proposed for deciding both real and fake news. Several traditional and mostly used Machine-Learning classification algorithms have been utilized to the given dataset of news for

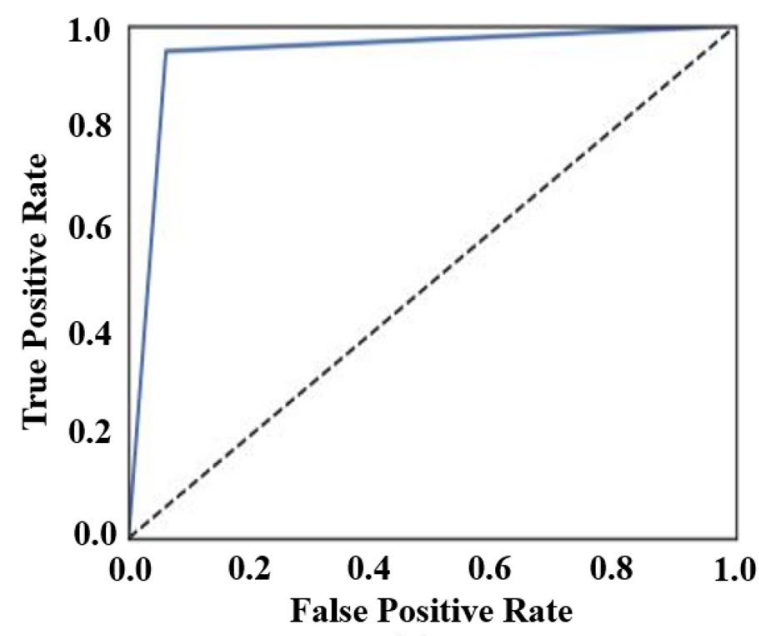

(a)

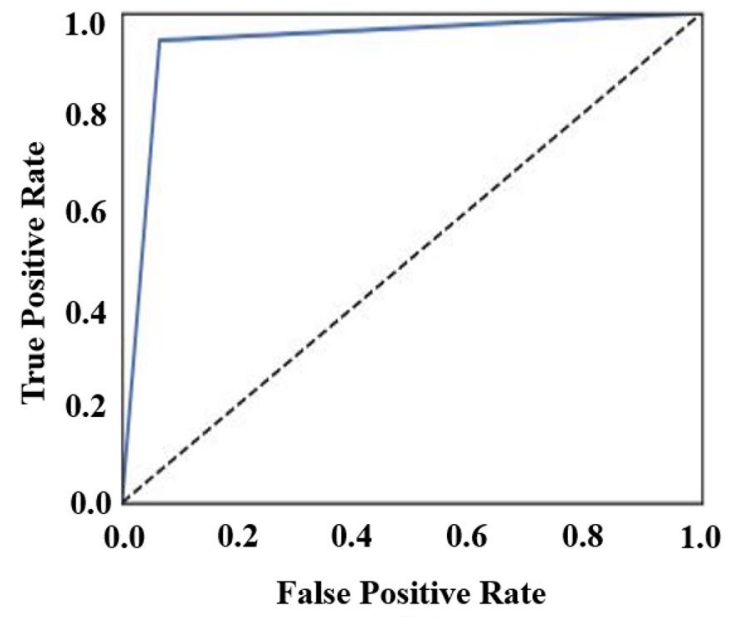

(b)

Fig. 5 ROC curve of voting classifier $\mathbf{a}$ soft vote and $\mathbf{b}$ hard vote

Table 2 Representation of Best Score, Accuracy, Precision, Recall and ROC Score after tuning MLP, X-Gradient Boosting and Logistic Regression

Table 3 Report for Ensemble Voting Classifier

\begin{tabular}{llllll}
\hline Classification type & Best score & Accuracy & Precision & Recall & ROC score \\
\hline MLP & 92.59 & 93.83 & 85.94 & 85.49 & 93.83 \\
X-Gradient Boosting & 92.92 & 92.87 & 86.67 & 87.67 & 92.87 \\
Logistic Regression & 93.03 & 98.21 & 85.44 & 90.01 & 93.03 \\
\hline
\end{tabular}

\begin{tabular}{lllllll}
\hline Voting type & Type & Test score & Precision & Recall & F1 & ROC score \\
\hline Soft & Real & 94.47 & 95 & 94 & 94 & 94.49 \\
& Fake & & 94 & 95 & 95 & \\
\multirow{4}{*}{ Hard } & Average & & 95 & 95 & 95 & \\
& Real & 93.99 & 94 & 94 & 94 & 93.98 \\
& Fake & & 94 & 94 & 94 & \\
& Average & & 94 & 94 & 94 & \\
\hline
\end{tabular}


masterminding them into real and fake. The results exhibited that this element would be astute to use regarding the accuracy, precision, recall, ROC score, F1 so as to control utilizing similar news aggregations and classification techniques. In addition, the results exhibited that Ensemble Voting classifier demonstrated better sufficiency scores when stood out from the results procured by the individual classifiers.

There are several interesting options for future work. One is to make use of other features available in the dataset like retweets, social networks, Instagram images community and learn highlights for the phony news discovery. Likewise, our proposed structure could be stretched out to identify fake news continuously as it is actualized in a streaming way. Deep learning techniques can also be implemented to improve accuracy and test scores.

\section{Conflict of interest}

The authors declare that they have no conflict of interest.

Funding This study was self-funded.

\section{References}

1. Bond CF Jr, DePaulo BM (2006) Accuracy of deception judgments. Personal Soc Psychol Rev 10(3):214-234

2. DePaulo BM, Lindsay JJ, Malone BE, Muhlenbruck L, Charlton K, Cooper H (2003) Cues to deception. Psychol Bull 129:74

3. Smith PK, Mahdavi J, Carvalho M, Fisher S, Russell S, Tippett $\mathrm{N}$ (2008) Cyberbullying: its nature and impact in secondary school pupils. J Child Psychol Psychiatry 49:376-385

4. Kowalski RM, Giumetti GW (2017) Bullying in the digital age. In: Martellozzo E, Jane EA (eds) Cybercrime and its victims. Routledge, Abingdon, pp 167-186

5. Krishnamurthy G, Majumder N, Poria S, Cambria E (2018) A deep learning approach for multimodal deception detection. ArXiv preprint arXiv:1803.00344

6. Wang Y, Ma F, Jin Z, Yuan Y, Xun G, Jha K, Su L, Gao J (2018) EANN: event adversarial neural networks for multi-modal fake news detection. In: Proceedings of the 24th ACM SIGKDD international conference on knowledge discovery and data mining, pp 849-857. ACM

7. Yuan D, Lu X, Li D, Liang Y, Zhang X (2019) Particle filter redetection for visual tracking via correlation filters. Multimed Tools Appl 78(11):14277-14301

8. Ou W, Yuan D, Liu Q, Cao Y (2018) Object tracking based on online representative sample selection via non-negative least square. Multimed Tools Appl 77(9):10569-10587

9. Ou W, Yuan D, Li D, Liu B, Xia D, Zeng W (2017) Patch-based visual tracking with online representative sample selection. J Electron Imaging 26(3):033006

10. Yuan D, Lu S, Li D, Zhang X (2019) Graph refining via iterative regularization framework. SN Appl Sci 1(5):387
11. Yuan D, Zhang X, Liu J, Li D (2019) A multiple feature fused model for visual object tracking via correlation filters. Multimed Tools Appl 78(19):27271-27290

12. Jin Z, Cao J, Jiang Y-G, Zhang Y (2014) News credibility evaluation on microblog with a hierarchical propagation model. In: 2014 IEEE international conference on data mining (ICDM). IEEE, pp 230-239

13. Shen H, Ma F, Zhang X, Zong L, Liu X, Liang W (2017) Discovering social spammers from multiple views. Neurocomputing 225(2017):49-57

14. Ahmed H, Traore I, Saad S (2017) Detection of online fake news using $\mathrm{N}$-gram analysis and machine learning techniques. In: International conference on intelligent, secure, and dependable systems in distributed and cloud environments. Springer, Cham

15. Shu K, Sliva A, Wang S, Tang J, Liu H (2017) Fake news detection on social media: a data mining perspective. ACM SIGKDD Explor Newsl 19(1):22-36

16. Shu, K, Wang S, Liu H (2017) Exploiting tri-relationship for fake news detection. ArXiv:1712.07709

17. Gilda S (2017) Evaluating machine learning algorithms for fake news detection. In: 2017 IEEE 15th student conference on research and development (SCOReD). IEEE

18. Wang WY (2017) "Liar, liar pants on fire": a new benchmark dataset for fake news detection. ArXiv:1705.00648

19. Ruchansky N, Seo S, Liu Y (2017) CSI: a hybrid deep model for fake news detection. In: Proceedings of the 2017 ACM on conference on information and knowledge management. ACM, pp 797-806

20. Shu K, Bernard HR, Liu H (2018) Studying fake news via network analysis: detection and mitigation. ArXiv:1804.10233

21. Shu K, Wang S, Liu H (2018) Understanding user profiles on social media for fake news detection. In: IEEE conference on multimedia information processing and retrieval (MIPR), pp 430-435

22. Mahabub A (2019) A robust voting approach for diabetes prediction using traditional machine learning techniques. SN Appl Sci 1(12):1667

23. Mahabub A, Mahmud MI, Hossain MF (2019) A robust system for message filtering using an ensemble machine learning supervised approach. ICIC Express Lett Part B Appl 10:805811. https://doi.org/10.24507/icicelb.10.09.805

24. Mahabub A, Habib AZSB (2019) A voting approach of modulation classification for wireless network. In: Proceedings of the 6th international conference on networking, systems and security, pp 133-138. ACM

25. Granik M, Mesyura V (2017) Fake news detection using naive Bayes classifier. In: 2017 IEEE first Ukraine conference on electrical and computer engineering (UKRCON). IEEE

26. Tretyakov K (2004) Machine learning techniques in spam filtering. Institite of Computer Science, University of Tartu, Tartu

27. Iyer A, Pandey A, Pamnani D, Pathak K, Hajgude J (2014) “Email Filtering and Analysis Using Classification Algorithms", IT Dept, VESIT. Int J Comput Sci Issues 11(4):1

28. Patidar V, Singh D, Singh A (2013) A novel technique of email classification for spam detection. Int J Appl Inf Syst 5(10):15-19

29. Annasaheb $A B$, Verma VK (2016) Data mining classification techniques: a recent survey. Int J Emerg Technol Eng Res 4(8):51-54

30. Shrivastava S, Anju R (2017) Spam mail detection through data mining techniques. In: 2017 International conference on intelligent communication and computational techniques (ICCT), pp 61-64. IEEE

31. Hastie T, Rosset S, Zhu Ji, Zou H (2009) Multi-class AdaBoost. Stat Interface 2(3):349-360 
32. Parviz M, Shahram Moin M (2008) Multivariate polynomials estimation based on gradientboost in multimodal biometrics. In: International conference on intelligent computing, pp 471-477. Springer, Berlin

33. Harrell FE (2015) Regression modeling strategies. Springer series in statistics, 2nd edn. Springer, New York
Publisher's Note Springer Nature remains neutral with regard to jurisdictional claims in published maps and institutional affiliations. 